As another important consideration, individual Notch ligand/receptor pairs may have unique effects on hematopoietic function. Shao et al. focus on signaling through the Notch1 receptor, which is the predominant receptor expressed in endothelial cells. However, both Notch1 and Notch2 are present in HSPC, and a specific role for Notch2 in HSPC differentiation following bone marrow injury has been reported. ${ }^{3}$ Recent advances in the biophysics of Notch signaling could provide explanations as to how engagement of distinct receptor-ligand pairs can lead to divergent functions. ${ }^{20}$ Nandagopal et al. showed that Dll1/Notch1 signaling induced pulsatile Notch activation whereas D114/Notch1 signaling resulted in sustained Notch activation during myogenesis, allowing for ligand discrimination. Additional differences in the signaling potential of specific ligand-receptor pairs may also exist. ${ }^{21}$ Whether similar biophysical and functional differences apply to the effects of individual Notch receptors in hematopoietic progenitors remains to be investigated.

Altogether, Shao et al. provide compelling data indicating that activation of Notch signaling between bone marrow endothelial cells is necessary for niche regeneration, as well as efficient and timely hematopoietic recovery after bone marrow injury. With a panoply of Notch receptors and ligands expressed throughout the bone marrow, Notch has the potential to regulate a number of communication channels between and among bone marrow cellular compartments. Future research should parse these cellular conversations to fully understand how Notch signaling helps to orchestrate hematopoiesis.

\section{References}

1. Crane GM, Jeffery E, Morrison SJ. Adult haematopoietic stem cell niches. Nat Rev Immunol. 2017;17(9):573-590.

2. Poulos MG, Guo P, Kofler NM, et al. Endothelial Jagged-1 is necessary for homeostatic and regenerative hematopoiesis. Cell Rep. 2013;4(5):1022-1034.

3. Varnum-Finney B, Halasz LM, Sun M, Gridley T, Radtke F, Bernstein ID. Notch2 governs the rate of generation of mouse long- and short- term repopulating stem cells. J Clin Invest. 2011;121(3):1207-1216.

4. Oh P, Lobry C, Gao J, et al. In vivo mapping of notch pathway activity in normal and stress hematopoiesis. Cell Stem Cell 2013;13(2):190-204.

5. Tikhonova AN, Dolgalev I, Hu H, et al. The bone marrow microenvironment at single-cell resolution. Nature. 2019;569(7755):222-228.

6. Butler JM, Nolan DJ, Vertes EL, et al. Endothelial cells are essential for the self-renewal and repopulation of Notch-dependent hematopoietic stem cells. Cell Stem Cell. 2010;6(3):251-264.

7. Klinakis A, Lobry C, Abdel-Wahab O, et al. A novel tumour-suppressor function for the Notch pathway in myeloid leukaemia. Nature. 2011;473(7346):230-233.

8. Maillard I, Koch U, Dumortier A, et al. Canonical notch signaling is dispensable for the maintenance of adult hematopoietic stem cells. Cell Stem Cell. 2008;2(4):356-366.

9. Duarte S, Woll PS, Buza-Vidas N, et al. Canonical Notch signaling is dispensable for adult steady-state and stress myelo-erythropoiesis. Blood. 2018;131(15):1712-1719.

10. Shao L, Sottoriva K, Palasiewicz K, et al. A Tie2-Notch1 signaling axis regulates regeneration of the endothelial bone marrow niche. Haematologica. 2019;104:(11):2164-2177.

11. Kopan R, Ilagan MX. The canonical Notch signaling pathway: unfolding the activation mechanism. Cell. 2009;137(2):216-233

12. Yu VW, Saez B, Cook C, et al. Specific bone cells produce DLL4 to generate thymus-seeding progenitors from bone marrow. J Exp Med. 2015;212(5):759-774

13. Guo P, Poulos MG, Palikuqi B, et al. Endothelial jagged-2 sustains hematopoietic stem and progenitor reconstitution after myelosuppression. J Clin Invest. 2017;127(12):4242-4256

14. Lee SU, Maeda M, Ishikawa Y, et al. LRF-mediated Dll4 repression in erythroblasts is necessary for hematopoietic stem cell maintenance. Blood. 2013;121(6):918-929.

15. Hooper AT, Butler JM, Nolan DJ, et al. Engraftment and reconstitution of hematopoiesis is dependent on VEGFR2-mediated regeneration of sinusoidal endothelial cells. Cell Stem Cell. 2009;4(3):263-274

16. Kopp HG, Avecilla ST, Hooper AT, et al. Tie2 activation contributes to hemangiogenic regeneration after myelosuppression. Blood. 2005;106(2):505-513

17. Wang L, Zhang H, Rodriguez S, et al. Notch-dependent repression of miR-155 in the bone marrow niche regulates hematopoiesis in an NF-kB-dependent manner. Cell Stem Cell. 2014;15(1):51-65.

18. Himburg HA, Termini CM, Schlussel L, et al. Distinct bone marrow sources of pleiotrophin control hematopoietic stem cell maintenance and regeneration. Cell Stem Cell. 2018;23(3):370-381.e5.

19. Tetzlaff F, Fischer A. Control of blood vessel formation by notch signaling. Adv Exp Med Biol. 2018;1066:319-338.

20. Nandagopal N, Santat LA, LeBon L, Sprinzak D, Bronner ME, Elowitz MB. Dynamic ligand discrimination in the notch signaling pathway. Cell. 2018;172(4):869-880.e19.

21. Tveriakhina L, Schuster-Gossler K, Jarrett SM, et al. The ectodomains determine ligand function in vivo and selectivity of DLL1 and DLL4 toward NOTCH1 and NOTCH2 in vitro. Elife. 2018;7.

\title{
Time for revival of the red blood cell count and red cell mass in the differential diagnosis between essential thrombocythemia and polycythemia vera?
}

\author{
Hans Carl Hasselbalch \\ Department of Hematology, Zealand University Hospital, Roskilde, Denmark \\ E-mail: HANS CARL HASSELBALCH - hans.hasselbalch@gmail.com
}

doi:10.3324/haematol.2019.229039

\section{Red blood cell indices, red blood cell mass and bone marrow biopsy in the differential diagnosis between essential thrombocythemia and polycythemia vera?}

The correct diagnostic classification of the Philadelphia-negative chronic myeloproliferative neoplasms (MPN) in the three subcategories, essential thrombocythemia (ET), polycythemia vera (PV) and primary myelofibrosis (PMF), relies upon diagnostic criteria that aim at minimizing misclassification. ${ }^{1}$ Several reports have addressed the issue that JAK2V617F positive "ET" patients are frequently misclassified since they actually have a diagnosis of $\mathrm{PV}^{2-8}$ This misclassification is partly based upon the use of the hemoglobin $(\mathrm{Hb})$ concentration as a surrogate marker for the red cell mass (RCM), irrespective of the fact that the $\mathrm{Hb}$ concentration is influenced by iron deficiency, which is prevalent in PV patients. Indeed, these concerns have been addressed and confirmed in several studies showing that a high proportion of ET patients (approx. 45-65\%) did not meet the World Health Organization (WHO) diagnostic criterion 
of an elevated $\mathrm{Hb}$, despite an increased Cr-51 RCM.5.7 However, despite the convincing data published in 2005 by Johansson et al., ${ }^{5}$ these concerns were not translated into the revised 2007 WHO diagnostic recommendations. These recommendations, therefore, remained unchanged $^{9}$ and were addressed and met by alternative diagnostic approaches in ET, PV and PMF patients. ${ }^{2}$ In their 2013 study, $^{3}$ Silver et al. for the first time prospec-

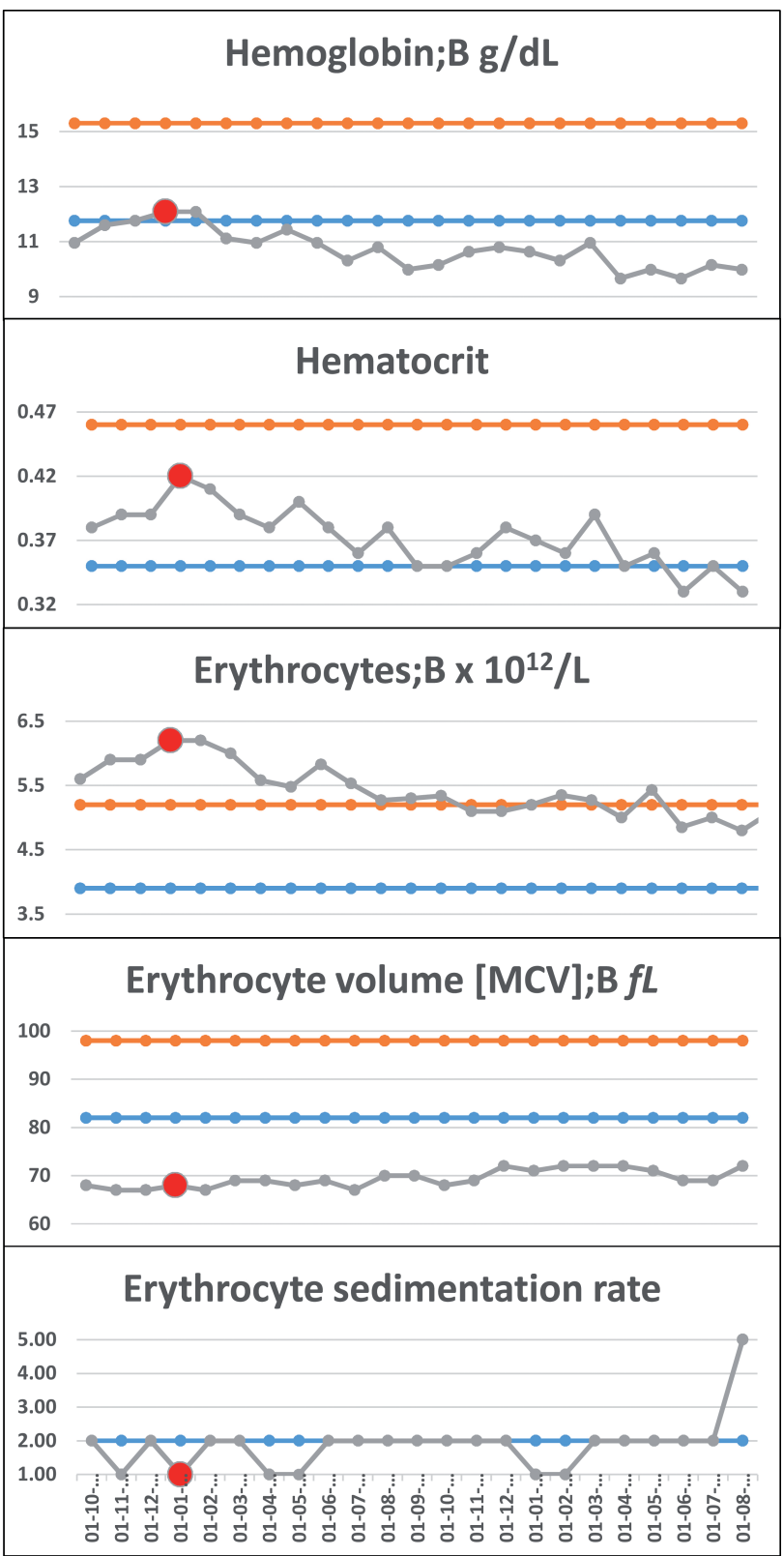

Figure 1. Association between the hemoglobin $(\mathrm{Hb})$-concentration, hematocrit, red blood cell (RBC) count and mean corpuscular volume (MCV) values in a 76year old woman with polycythemia vera. The Figure illustrates that: (i) the RBC count is a a more accurate indicator of erythrocytosis than the Hb-concentration and the hematocrit; (ii) that this dissociation is consequent to iron deficiency as evidenced by a lowered MCV; and (iii) the hyperviscosity state due to the raised $\mathrm{RBC}$ count is reflected in a low erythrocyte sedimentation rate (SR) $(<1$ or 2 $\mathrm{mm} / \mathrm{h}$ ) (normal range: $2-20 \mathrm{~mm} / \mathrm{h}$ ). The need for phlebotomies in this patient was monitored by the elevated RBC count and the lowered SR, and tightly associated with the emergence of headache, which immediately resolved after phlebotomy. tively evaluated the accuracy of the 2007 WHO criteria for diagnosing PV, especially in "early-stage" patients. This and other studies support the latest updated WHO criteria (2016) for diagnosing MPN, ${ }^{1,9}$ which included these novel data with regard to the inaccuracy of the $\mathrm{Hb}$ concentration, and even the hematocrit (HCT), in the differential diagnosis between ET and PV patients by lowering the $\mathrm{Hb} / \mathrm{HCT}$ thresholds (> $16.5 \mathrm{~g} / \mathrm{dL} / 0.49$ in men and $>16 \mathrm{~g} / \mathrm{dL} / 0.48$ in women). ${ }^{1}$ Thus, the 2013 Silver study in a prospective setting and with a median 5-year follow up time convincingly demonstrated that the surrogate markers $\mathrm{Hb}$ and $\mathrm{HCT}$ are inadequate in the assessment of an increased RCM for early PV cases, since 64.3\%, 28.5\%, and $28.5 \%$ of their patients would not have been diagnosed as PV using $\mathrm{Hb}, \mathrm{HCT}$, and either $\mathrm{Hb}$ or HCT values, respectively. ${ }^{3}$ Importantly, of the 28 patients with an increased RCM in their study, 18 did not meet the WHO 2007 criteria for an increased $\mathrm{Hb}$ value. For the four women, the median $\mathrm{Hb}$ count was $15.2 \mathrm{~g} / \mathrm{dL}$ (range: 14.4$16.4 \mathrm{~g} / \mathrm{dL}$ ) and for the 14 men $17.2 \mathrm{~g} / \mathrm{dL}$ (range: 15.6-18.1 $\mathrm{g} / \mathrm{dL}$ ), respectively. Similarly, eight patients (1 woman and 7 men) did not meet the WHO criteria for an increased HCT value, being $44.3 \%$ for the woman, and for the seven men the median HCT was $48.5 \%$ (range: 45.7-49.4\%). ${ }^{3}$ Silver et al. also highlighted the value of bone marrow (BM) morphology ${ }^{3}$ as emphasized in the WHO classification. Accordingly, this study supported previous reports by Johansson et al. ${ }^{5}$ Cassinat et al.,${ }^{6}$ and Alvarez-Larran et al. ${ }^{7}$ which all revived ancient knowledge, written by the Polycythemia Vera Study Group (PVSG), and underscoring the inaccuracy of the $\mathrm{Hb}$ and the HCT values for diagnosing PV and the need for RCM measurement instead. ${ }^{10}$ This has since fostered intense debate in several reviews and perspective papers expressing conflicting opinions. On the one hand, some authors believe that only RCM measurements can reliably distinguish PV from other MPN, ${ }^{2-4,11}$ while others would disregard RCM measurements, ${ }^{12-15}$ arguing that $\mathrm{Hb} / \mathrm{HCT}$ thresholds should be used as surrogate markers for RCM measurements. This lively debate has recently been further fueled by a comprehensive and scholarly review on $\mathrm{MPN}$, emphasizing the urgent need for RCM investigations to distinguish PV from other MPN, ${ }^{16}$ adding that BM morphology has no place in the distinction of PV from other MPN subtypes. ${ }^{16}$ Others have highlighted the importance of BM morphology in PV and its usefulness in distinguishing between ET and PV.,17-21 A very recent study has established a clear-cut distinction between ET and PV, and, therefore, also the reproducibility of BM morphology in so-called masked polycythemia vera (mPV) and its differentiation from ET. ${ }^{21}$ The disease entity $\mathrm{mPV}$ will be further addressed below.

\section{Do the revised and lowered thresholds for Hb/HCT} levels unmask undiagnosed PV patients in the general population when "potential PV patients" are being referred?

In 2014, the issue as to which of the three red cell parameters, Hb, HCT or RCM, to use as the diagnostic hallmark of PV was thoroughly reviewed by Barbui et al. They also critically addressed the validity and applicability of the three major diagnostic classification systems for 
PV as proposed by the PVSG, the British Committee for Standards in Hematology (BCSH), and the WHO. ${ }^{13}$ It was suggested either to reduce the thresholds for the Hb-concentration, or to include the HCT as a major diagnostic criterion in association with the JAK2V617F mutation. In this review, the existence of prodromal (latent PV-disease), named "masked" PV and defined by Barbui et al., ${ }^{13}$ was also addressed, covering patients not meeting the required $\mathrm{Hb}$ or $\mathrm{HCT}$ threshold levels as defined in the WHO and BCSH criteria. ${ }^{22,23}$ With regards to the impact of lowering the $\mathrm{Hb}$ and the HCT thresholds for PV, influenced by the above studies, ${ }^{3,5-8,82,223}$ the 2016 revised WHO criteria have not resolved the conflicting opinions, ${ }^{24,25}$ as recently addressed and discussed in depth. ${ }^{26,27}$ However, very interestingly, applying the lower $\mathrm{Hb}$ thresholds as reported by the WHO 2016 criteria in the Canadian population, Ethier et al. found an $\mathrm{Hb}$ value at or above the threshold in $4.1 \%$ of all complete blood counts from unselected males and in $0.35 \%$ of females. These figures increased the incidence of "potential PV patients" by up to 12 -fold in males and 3-fold in females. The same pattern was demonstrated when including the neutrophil and platelet count, implying that up to 60 times more males and three times more women would be suspected of suffering from MPN and would accordingly require diagnostic investigations. ${ }^{24}$ According to the screening procedure in clinical practice as described by Rumi and Cazzola, ${ }^{25}$ the best compromise between the need for an early diagnosis of PV patients and the risk of excessively expanding the number of potential PV patients would be a threshold of $17 \mathrm{~g} / \mathrm{dL}$ in men. Applying this $\mathrm{Hb}$ cut-off value, Barbui et al. found that $14 \%$ of their 375 patients presenting with WHO-defined PV did not meet the 2016 criteria. ${ }^{26}$ In the context of screening procedures in clinical practice and which blood cell counts to use, according to the Canadian data, the Hb/HCT thresholds as defined in the 2016 WHO criteria will markedly increase the number of individuals with suspected PV in the general population. ${ }^{24}$ Given this, it is important to note that a very recent Danish study has found MPN to be massively underdiagnosed with an estimate of 10,000 undiagnosed MPN in Denmark, corresponding to approximately 550,000 US citizens having an undiagnosed MPN and accordingly being at a considerable risk of thrombosis. ${ }^{28}$ In this perspective, it might be much more cost-effective to screen high-risk MPN individuals and obtain a diagnosis earlier rather than later when the individual has already suffered one or more potentially life-invalidating thrombotic events before being diagnosed with ET or $\mathrm{PV}^{28}$

\section{Consequences of misclassification of PV as ET}

There are several consequences of misclassifying PV patients as ET.

1) Misclassification of JAK2V617F positive ET patients as ET instead of PV implies that these patients are not phlebotomized and are, therefore, exposed to an increased risk of potentially life-invalidating or life-threatening thrombotic complications due to the increased RCM. This is not a trivial risk, in particular in those patients aged $<60$ years without a prior thrombosis but with leukocytosis and platelet counts $<1500 \times 10^{9} / \mathrm{L}$, since these patients are categorized as "low-risk" accord- ing to international guidelines and are not offered cytoreductive treatment, irrespective of the fact that both leukocytes and platelets are deeply involved in the development of atherothrombosis, ${ }^{29-33}$ the JAK2V617F mutation promotes atherothrombosis, being associated with e.g. transitory cerebral ischemia, completed stroke and ischemic heart disease, and leukocytosis per se is considered a risk factor for thrombosis in the background population, ${ }^{34}$ and a causative factor for thrombosis in ET and PV patients. ${ }^{35}$ Importantly, the most recent studies, including a meta analysis study, have provided evidence that leukocytosis is a risk factor for thrombosis in the MPN-population as well. 36,37

2) Misclassifying JAK2V617F positive "ET" patients as "ET" instead of PV has a huge impact on any prognostic model that compares the prognosis of ET and PV patients, the potential outcome being that JAK2V617F positive ET patients have an inferior prognosis as compared to those who are JAK2V617F negative or CALR-positive. ${ }^{38}$ In several published studies these differences might be explained by the fact that PV patients - not being phlebotomized - have been included in the ET-cohorts. This may also hold true for mPV patients who have an increased risk of thrombosis (young patients) ${ }^{39}$ and poorer survival than PV patients. This is likely explained by the fact that several mPV patients have not been phlebotomized despite an expanded RCM.

3) Results from studies on safety and efficacy of any drug, both those conventionally used [e.g. hydroxyurea $(\mathrm{HU})$, interferon- $\alpha 2$ (IFN) and anagrelide] and novel agents such as ruxolitinib or experimental drugs (in clinical trials for future approval for the indication of ET or PV) may be severely undermined, impossible to interpret, and therefore not credible.

4) Building future therapeutic recommendations and prognostic models on a diagnostic platform that does not take into account the true nature of a disease (e.g. a higher rate of thrombosis in PV than ET, a higher rate of transformation to myelofibrosis and acute myelogenous leukemia in PV than in ET, a reduced life expectancy in PV as compared to ET) due to diagnostic misclassification undermines our current understanding and concepts on MPN in highly important issues. These include pathogenetic mechanisms for disease evolution both in terms of molecular phenotypes, clinical phenotypes and associations between them, diagnostic classification in the biological continuum from early cancer stages (ET, PV) to the advanced myelofibrosis stage, and, not least, when and how to treat MPN. The cornerstone treatment of PV is phlebotomies, carried out to alleviate the hyperviscosity state due to an expanded RCM and thereby to reduce the risk of the deadly thrombosis seen in median survival figures for PV patients of 18 months without such treatment. This approach may, however, be misguided by using only the $\mathrm{Hb}$ concentration and the HCT as these are profoundly influenced by the iron-deficient state in patients with PV and also in JAK2V617F positive ET patients in whom erythropoietin (Epo) and ferritin levels and the mean corpuscular volume (MCV) values have been repeatedly reported to be lower than in JAK2V617F negative ET patients. ${ }^{40}$

5) Without an estimation of RCM and plasma volume 
in the diagnostic setting of JAK2V617F positive ET and $\mathrm{PV}$, the transitional stage between ET and PV may be wrongly described as a new disease entity within MPN. ${ }^{22}$ Thus, it is tempting to speculate whether the "novel" disease entity (mPV) would ever have been born, if arguing that a large proportion of these $\mathrm{mPV}$ patients are only "masked" as long as RCM is not being estimated. As discussed above, reports on masked $\mathrm{PV}^{22,23}$ were influential in lowering the $\mathrm{Hb} / \mathrm{HCT}$ thresholds in the 2016 WHO classification of MPN.,1,27 This was defined as a new JAK2V617F-positive entity with a phenotype mimicking ET (isolated thrombocytosis) but, as in PV, associated to endogenous erythroid colony formation (EEC) or the BM features of PV, which had previously been described as latent or inapparent PV. ${ }^{41,42}$ The revised WHO 2016 classification was, among others, based upon the MPV studies, which defined threshold values as optimal cut-off levels for distinguishing JAK2V617F ET from mPV ( $\mathrm{Hb} 16.5$ $\mathrm{g} / \mathrm{dL} / \mathrm{HCT} 0.49$ in men and $16 \mathrm{~g} / \mathrm{dL} / \mathrm{HCT} 48 \%$ in women, respectively $)^{43}$ and were subsequently validated in larger cohort studies. ${ }^{15,44}$

Very recently, the notion that mPV may not be a novel disease entity but has emerged consequent to the inaccuracy of diagnosing $\mathrm{PV}$ in the absence of an estimation of RCM has been supported by a large French single center study of 2,480 RCM estimations in patients with JAK2V617F positive ET, "masked PV", and PV. ${ }^{45}$ This study showed that patients with mPV actually have an increased RCM and are, therefore, easily "unmasked " and revealed to be true PV once RCM is estimated. ${ }^{45}$ Thus, these $\mathrm{mPV}$ patients share clinical and biological features with both ET and PV, with a median age, platelet, $\mathrm{Hb}$ and leukocyte levels comparable to those of ET patients, and at the same time classic PV features, (which, in addition to the increased RCM, also include lower Epo level and lower MCV), and have splenomegaly more frequently than ET patients. ${ }^{45}$ Accordingly, patients being described as $\mathrm{mPV}$ nicely present a picture of a diagnosis of MPN as a moving target that is highly dependent on the time point for diagnosis in the biological continuum from early JAK2V617F positive ET to overt PV.

The $\mathrm{mPV}$ story underscores the urgent need for a renaissance of the RCM and plasma volume assessment in these patients, since otherwise JAK2V617F positive "ET" patients and $\mathrm{mPV}$ patients will not receive adequate treatment by phlebotomies and so will obviously also have an increased risk of thrombosis. ${ }^{3,39}$ The French and other studies, including those pioneered by Silver and Spivak, also put into perspective the view that it is indeed possible to incorporate RCM estimations into 'good clinical practice' in the differential diagnosis between JAK2V617F positive ET and PV. ${ }^{2-8,16,645}$ However, as noted above, there are still conflicting opinions as to the need for RCM measurements in distinguishing between patients with JAK2V617F positive ET, $\mathrm{mPV}$ and overt $\mathrm{PV}^{2 \cdot-4,11-15}$ Of note, a very recent study showed that when applying the 2016 WHO criteria, increased RCM was significantly associated with increased $\mathrm{Hb} / \mathrm{HCT}(93.8 \% / 94.6 \%),{ }^{15}$ thus supporting the $2016 \mathrm{WHO}$ criteria for $\mathrm{PV}$, implying $\mathrm{Hb} / \mathrm{HCT}$ values should be used as surrogate markers for RCM measurements. ${ }^{15}$ In this study, the importance of BM morphology for a diagnosis of PV was also highlighted. ${ }^{15}$

\section{Red blood cell count as a surrogate marker for red cell mass?}

Recently, Michiels et al. underscored the importance of RBC count in addition to a BM biopsy as a powerful tool to differentiate between ET and PV. ${ }^{8}$ In their study, the diagnostic value of RCM in relation to RBC count, $\mathrm{Hb}$ and HCT in discriminating between JAK2V617F ET and PV was assessed. The best correlation was found between RBC count and RCM. Thus, at RCM above 30 $\mathrm{mL} / \mathrm{kg}$ the $\mathrm{RBC}$ count was above $5.8 \times 10^{12} / \mathrm{L}$, and this diagnosed PV in all their patients. All JAK2V617F ET patients had a normal RCM and a RBC count below $5.8 \times 10^{12} / \mathrm{L}$. It was concluded that a RBC count within the normal range $\left(<5.8 \times 10^{12} / \mathrm{L}\right.$ in males and $<5.6 \times 10^{12} / \mathrm{L}$ in females) enables JAK2V617F ET to be distinguished from prodromal PV and overt PV. Thus, they also concluded that the $\mathrm{RBC}$ count and a BM biopsy might obviate the need for RCM measurement. ${ }^{8}$

\section{Are hemoglobin and hematocrit values imperfect surrogate markers for red cell mass?}

In this issue of Haematologica, Silver et al. convincingly confirm the urgent need to investigate JAK2V617F positive ET patients using RCM estimations ${ }^{46}{ }^{46}$ repeating the important message that a normal $\mathrm{Hb}$ or HCT value does not signify a normal RCM in MPN. ${ }^{2-8,10,11,16}$ Based upon JAK2V617F positivity, chromium-51 RCM, and BM biopsy morphology, 83 and 39 patients were diagnosed with PV and ET, respectively. Chromium-51 RCM separated PV from ET JAK2V617F, whereas red cell values $(\mathrm{Hb}$, HCT, RBC count) overlapped in $25.0-54.7 \%$. The authors concluded that a significant proportion of PV patients may be underdiagnosed by using only red cell values. Of note, using ROC analyses, the authors found threshold values for the $\mathrm{Hb} / \mathrm{HCT}$ coincidentally similar to the WHO 2016 criteria. Furthermore, it was concluded that (without isotope studies) BM biopsies and serum erythropoietin values should become mandatory since they improve diagnostic accuracy. In this perspective, the paper by Silver et al. is highly relevant and timely. It carries a novel approach into the future and will hopefully promote an optimal classification of MPN by a renaissance of the use of RCM estimations (the "gold standard" for discriminating JAK2V617F ET from PV) as a highly important tool to ensure a correct diagnostic classification of MPN. This will be a major scientific step forward in improving good clinical practice in MPN patients. It will reintroduce RCM and plasma volume estimations as essential for correct diagnostic classification of MPN, at least in JAK2V617F positive "ET" patients. In patients with huge splenomegaly but still a low normal or even lowered HCT, in the transitional stages between PV and post-polycythemic myelofibrosis, a RCM estimation may reveal the true nature of the disease as PV and accordingly a need for phlebotomies due to an expanded RCM in patients who still present a normal $\mathrm{Hb}$-concentration and normal HCT consequent to hemodilution due to an expanded plasma volume. As previously noted, RCM estimation in JAK2V617F positive ET patients will likely reduce the risk of thrombosis in a substantial proportion of "ET" patients since they will be correctly classified as PV and will, therefore, receive treatment with phle- 
botomies. Additionally, these patients will then also have the opportunity to be treated with IFN in cases in which the institution does not include ET patients as an indication for being treated with IFN but does so if the patient has PV. This will be even more important when the new IFN-drug, ropeginterferon $\alpha-2 b$, has been licensed for use in newly diagnosed PV in Europe, and hopefully soon in the US as well. ${ }^{47}$ Based upon the above considerations, the paper by Silver et al. is also of utmost importance since by diagnosing many more JAK2V617F ET patients correctly as $\mathrm{PV}$, their findings may offer more patients the opportunity to be treated with IFN, which is the only agent that has shown to be disease modifying. In fact, in a subset of patients with early MPN disease (ET and PV), after approximately five years of IFN therapy it was seen that this approach may induce minimal residual disease (MRD), with normal cell counts, normal spleen size, a normal BM, and no detectable JAK2V617F mutation, representing an $M R D$ stage that may even be sustained after interrupting IFN for up to three years. ${ }^{48}$

Considering the findings by Silver et al. and Michiels et al., in some of their previous papers the RBC count is the most valuable parameter and is better than $\mathrm{Hb}$-concentration and hematocrit when considering the equation: $\mathrm{HCT}=\mathrm{RBC}$ count $\mathrm{xMCV}$, and taking into account that several PV patients have lowered MCV which accordingly lowers the HCT and wrongly dictates that a phlebotomy is not needed, irrespective of the fact that the RBC count is increased ${ }^{49}$ (Figure 1). In this context, it is also important to note that erythrocytosis in PV usually induces plasma volume expansion ${ }^{4,16,50}$ which may mask the true HCT, implying that the HCT in many PV patients, especially women, appears to be normal. ${ }^{2,4,16,41,50}$

These considerations are not only relevant at the time of diagnosis but also during the course of PV when several patients may not be phlebotomized when only using HCT and elevated RBC count is not taken into consideration (Figure 1). On the contrary, hydroxyurea (HU)-treated patients may be unnecessarily phlebotomized due to an HU-induced increase in MCV and accordingly also an increase in the HCT, although the RBC count is normal.

Hopefully, based upon previous reports on the need of the RCM in the diagnosis of MPN, ${ }^{2-7,10,11,1,6,49,50}$ and the most recent studies by Silver et al. and the French study discussed above, consideration of the RCM will be revived at many more MPN centers worldwide. Such efforts are not only expected to improve quality of life of the large proportion of undiagnosed PV patients amongst JAK2V617F positive ET patients, but likely prognosis as well, since they will be correctly diagnosed as PV and accordingly receive the cornerstone treatment of PV (phlebotomy) to reduce the HCT $<0.42$ in women and $<0.45$ in men. ${ }^{2-4,11,16,50 \cdot 53}$

The important distinction between different HCT levels for women and men when deciding the need for phlebotomies has been addressed in several papers. ${ }^{2,4,16,50.53}$ The rationales for this distinction are several and obvious, including the simple fact that women and men have different red blood cell volumes as reflected in different ranges for red cell indices. This common knowledge dictates that a woman's normal RCM is approximately 600 $\mathrm{mL}$ lower than that for men., ${ }^{4,53}$ Accordingly, a female patient with a hematocrit of $45 \%$ has at least an excess of approximately $600 \mathrm{~mL}$ blood $^{4}$ which associates with an increased risk of major thrombosis. ${ }^{54}$ Indeed, the study by Marchioli et al. clearly showed that allowing HCT between 0.45 and 0.50 is associated with a significant risk of death from cardiovascular causes or major thrombotic events $^{54}$ - also in the general population. ${ }^{55}$ These are lessons that we learnt from Pearson 30 years $\mathrm{ago}^{51,52}$ and which have been repeated since then in several other studies: an elevated HCT is associated with an increased risk of thrombosis. In fact, in the general population the risk of thrombosis at elevated HCT values has previously been reported to be driven by smoking, ${ }^{56}$ which has recently been associated with an increased risk of MPN. ${ }^{57}$

The excess blood volume is even larger in PV-patients with hepatic vein thrombosis, ${ }^{16}$ who often have a normal HCT due to an expanded plasma volume. ${ }^{4}$ Importantly, the thrombosis risk in JAK2V617F positive "ET" patients will likely be markedly reduced simply due to normalization of the expanded RCM by phlebotomies. Additionally, without a RCM estimation, some patients with JAK2V617F positive "ET" may be erroneously classified as "early prefibrotic myelofibrosis" while actually having undiagnosed PV for several years and then being referred with an enlarged spleen, a normal $\mathrm{Hb}$-concentration and a normal HCT, red cell values that are in the normal range due to hemodilution consequent to the expanded plasma volume associated with the enlarged spleen. Accordingly, in such patients, a RCM estimation may reveal an expanded RCM requiring phlebotomies to omit thrombotic complications, often at unusual sites such as portal thrombosis, mesenteric thrombosis and thrombosis of hepatic veins. ${ }^{58}$ Indeed, similar to $\mathrm{mPV}$ as a transitional stage in the biological continuum from ET to overt PV, one might speculate as to whether a proportion of JAK2V617F positive patients with a normal $\mathrm{Hb} / \mathrm{HCT}$ and splenomegaly classified as "early prefibrotic myelofibrosis" may actually have PV with an expanded RCM and expanded plasma volume in a transitional stage towards classic myelofibrosis.

Today, we still need to go over the important lessons from the history of MPN. Back in 1908, Osler taught us that the $\mathrm{RBC}$ count is superior to the $\mathrm{Hb}$ concentration as an indicator of erythrocytosis. ${ }^{2,4,16,50,59,60}$ This should, therefore, be used in the diagnosis of PV, and the lesson from the PV study group and from several authorities thereafter is that the RCM is "the gold standard" for an accurate diagnosis of $\mathrm{PV}$ in patients with $\mathrm{mPV}$ and its precursor stage: JAK2V617F positive ET.-8,16 In the paper by Silver et al. in this issue of Haematologica and in other papers, the importance of these lessons have been repeatedly highlighted. These will hopefully stimulate research into MPN towards additional comparative and correlative studies on the value of RCM estimations, the RBC count, and BM morphology in the diagnosis of MPN. Such studies are even more urgent taking into consideration a most recent review challenging and critically discussing the role of the hematocrit as a determinant risk factor for thrombosis in erythrocytosis. ${ }^{61}$ It is to be hoped that such studies may promote a consensus amongst MPN experts that the RCM is essential for a correct classification of JAK2V617F positive ET patients, $\mathrm{mPV}$ and PV patients. 
This will ensure timely treatment with phlebotomies in those patients who otherwise will be classified as ET and who would then carry an increased risk of potentially life-threatening or life-invalidating thrombotic complications. Future studies should also address whether the RBC count in addition to the erythrocyte SR may be simple but highly robust and reproducible indicators of an increased RCM and the hyperviscosity state, respectively, to be used in the diagnosis of PV and when monitoring PV patients for the need for phlebotomy. ${ }^{62}$

\section{References}

1. Arber DA, Orazi A, Hasserjian R, et al. The 2016 revision to the World Health Organization classification of myeloid neoplasms and acute leukemia. Blood. 2016;127(20):2391-2405.

2. Spivak JL, Silver RT. The revised World Health Organization diagnostic criteria for polycythemia vera, essential thrombocytosis, and primary myelofibrosis: an alternative proposal. Blood. 2008;112(2): 231-239

3. Silver RT, Chow W, Orazi A, Arles SP, Goldsmith SJ. Evaluation of WHO criteria for diagnosis of polycythemia vera: a prospective analysis. Blood. 2013;122(11):1881-1886.

4. Spivak JL. How I treat polycythemia vera. Blood. 2019;134(4):341352 .

5. Johansson PL, Safai-Kutti S, Kutti J. An elevated venous haemoglobin concentration cannot be used as a surrogate marker for absolute erythrocytosis: a study of patients with polycythaemia vera and apparent polycythaemia. Br J Haematol. 2005;129(5):701-705.

6. Cassinat B, Laguillier C, Gardin C, et al; PV-Nord Group. Classification of myeloproliferative disorders in the JAK2 era: is there a role for red cell mass? Leukemia. 2008;22(2):452-453.

7. Alvarez-Larran A, Ancochea A, Angona A, et al. Red cell mass measurement in patients with clinically suspected diagnosis of polycythemia vera or essential thrombocythemia. Haematologica. 2012;97(11):1704-1707.

8. Michiels JJ, Medinger M, Raeve HD, et al. Increased Erythrocyte Count on Top of Bone Marrow Histology but not Serum EPO Level or JAK2 Mutation Load Discriminates between JAK2V617F Mutated Essential Thrombocythemia and Polycythemia Vera. J Hematol Thromb Dis. 2015;3:S1-001.

9. Tefferi A, Thiele J, Orazi A, et al. Proposals and rationale for revision of the World Health Organization diagnostic criteria for polycythemia vera, essential thrombocythemia, and primary myelofibrosis: recommendations from an ad hoc international expert panel. Blood. 2007;110(4):1092-1097

10. Najean Y, Dresch C, Rain J, Chomienne C. Radioisotope investigations for the diagnosis and follow-up of polycythemic patients. In: Wasserman LR, Berk PD, Berlin NI, eds. Polycythemia Vera and the Myeloproliferative Disorders. Philadelphia, PA: Saunders; 1995:7990.

11. Spivak JL. Polycythemia vera: myths, mechanisms, and management. Blood. 2002;100(13):4272-4290.

12. Tefferi A. The rise and fall of red cell mass measurement in polycythemia vera. Curr Hematol Rep. 2005;4(4):213-217

13. Barbui T, Thiele I, Vannucchi AM, Tefferi A. Rethinking the diagnostic criteria of polycythemia vera. Leukemia. 2014;28(6):1191-1195.

14. Tefferi A, Barbui T. Polycythemia vera and essential thrombocythemia: 2017 update on diagnosis, risk-stratification, and management. Am J Hematol. 2017;92(1):94-108

15. Jakovic L, Gotic M, Gisslinger $\mathrm{H}$, et al. The WHO diagnostic criteria for polycythemia vera-role of red cell mass versus hemoglobin/hematocrit level and morphology. Ann Hematol. 2018;97(9):1581-1590.

16. Spivak JL. Myeloproliferative Neoplasms. N Engl J Med. 2017;376 (22):2168-2181.

17. Thiele J, Kvasnicka HM. Diagnostic impact of bone marrow histopathology in polycythemia vera (PV). Histol Histopathol. 2005; 20(1):317-328.

18. Kvasnicka HM. WHO classification of myeloproliferative neoplasms (MPN): a critical update. Curr Hematol Malig Rep. 2013;8(4):333341.

19. Madelung AB, Bondo H, Stamp I, et al. World Health Organizationdefined classification of myeloproliferative neoplasms: morphological reproducibility and clinical correlations-the Danish experience. Am J Hematol. 2013;88(12):1012-1016.
20. Gianelli U, Iurlo A, Cattaneo D, Lambertenghi-Deliliers G Cooperation between pathologists and clinicians allows a better diagnosis of Philadelphia chromosome-negative myeloproliferative neoplasms. Expert Rev Hematol. 2014;7(2):255-264.

21. Kvasnicka HM, Orazi A, Thiele J, et al. European leukemia net study on the reproducibility of bone marrow features in masked polycythemia vera and differentiation from essential thrombocythemia. Am J Hematol. 2017;92(10):1062-1067

22. Barbui T, Thiele J, Gisslinger $\mathrm{H}$, et al. Masked polycythemia vera (mPV): results of an international study. Am J Hematol. 2014;89(1):52-54

23. Barbui T, Thiele J, Carobbio A, et al. Masked polycythemia vera diagnosed according to $\mathrm{WHO}$ and $\mathrm{BCSH}$ classification. Am Hematol. 2014;89(2):199-202.

24. Ethier V, Sirhan S, Olney HJ, et al. The 2016 WHO criteria for the diagnosis of polycythemia vera: benefits and potential risks. [e-letters]. Blood. 2016. Available at: http://www.bloodjournal.org/content/127/20/2391/tab-e-letters.

25. Rumi E, Cazzola M. Diagnosis, risk stratification, and response evaluation in classical myeloproliferative neoplasms. Blood. 2017;129(6):680-692.

26. Barbui $\mathrm{T}$, Thiele J, Gisslinger $\mathrm{H}$, et al. Diagnostic impact of the revised WHO criteria for polycythemia vera. Am J Hematol. 2017;92(5):417-419.

27. Barbui T, Thiele J, Gisslinger $\mathrm{H}$, et al. The 2016 WHO classification and diagnostic criteria for myeloproliferative neoplasms: document summary and in-depth discussion. Blood Cancer J. 2018;8(2):15

28. Cordua S, Kjaer L, Skov V, et al. Prevalence and phenotypes of JAK2 V617F and calreticulin mutations in a Danish general population. Blood. 2019;134(5):469-479.

29. Ross R. Atherosclerosis - an inflammatory disease. N Engl J Med. 1999;340(2):115-126.

30. Davì G, Patrono C. Platelet activation and atherothrombosis. N Engl J Med. 2007;357(24):2482-2494.

31. Totani L, Evangelista V. Platelet-leukocyte interactions in cardiovascular disease and beyond. Arterioscler Thromb Vasc Biol. 2010;30(12):2357-2361.

32. Hoogeveen RM, Nahrendorf M, Riksen NP, et al. Monocyte and haematopoietic progenitor reprogramming as common mechanism underlying chronic inflammatory and cardiovascular diseases. Eur Heart J. 2018;39(38):3521-3527

33. Nahrendorf M. Myeloid cell contributions to cardiovascular health and disease. Nat Med. 2018;24(6):711-720

34. Coller BS. Leukocytosis and ischemic vascular disease morbidity and mortality: is it time to intervene? Arterioscler Thromb Vasc Biol. 2005;25(4):658-670.

35. Barbui T, Carobbio A, Rambaldi A, Finazzi G. Perspectives on thrombosis in essential thrombocythemia and polycythemia vera: is leukocytosis a causative factor? Blood. 2009;114(4):759-763.

36. Buxhofer-Ausch V, Steurer M, Sormann S, et al. Impact of white blood cells on thrombotic risk in patients with optimized platelet count in essential thrombocythemia. Eur J Haematol. 2018 Mar 30. [Epub ahead of print]

37. Carobbio A, Ferrari A, Masciulli A, Ghirardi A, Barosi G, Barbui T Leukocytosis and thrombosis in essential thrombocythemia and polycythemia vera: a systematic review and meta-analysis. Blood Adv. 2019;3(11):1729-1737.

38. Rumi E, Pietra D, Ferretti V, et al. JAK2 or CALR mutation status defines subtypes of essential thrombocythemia with substantially different clinical course and outcomes. Blood. 2014;123(10):1544 1551.

39. Lussana, F, Carobbio A, Randi ML, et al. A lower intensity of treatment may underlie the increased risk of thrombosis in young patients with masked polycythemia vera. Br J Haematol. 2014;2014;167(4):541-546

40. Campbell PJ, Scott LM, Buck G, et al. ; United Kingdom Myeloproliferative Disorders Study Group; Medical Research Council Adult Leukaemia Working Party; Australasian Leukaemia and Lymphoma Group. Definition of subtypes of essential thrombocythaemia and relation to polycythaemia vera based on JAK2 V617F mutation status: a prospective study. Lancet. 2005;366(9501):19451953.

41. Lamy $T$, Devillers A, Bernard M, et al. Inapparent polycythemia vera: an unrecognized diagnosis. Am J Med. 1997;102(1):14-20.

42. Thiele J, Kvasnicka HM, Diehl V. Initial (latent) polycythemia vera with thrombocytosis mimicking essential thrombocythemia. Acta Haematol. 2005;113(4):213-219.

43. Barbui T, Thiele J, Carobbio A, et al. Discriminating between essential thrombocythemia and masked polycythemia vera in JAK2 mutated patients. Am J Hematol. 2014;89(6):588-590.

44. Barbui T, Thiele J, Kvasnicka HM, et al. Essential thrombocythemia 
with high hemoglobin levels according to the revised WHO classification. Leukemia. 2014;28(10):2092-2094.

45. Maslah N, Soret J, Dosquet C Sr, et al. Masked polycythemia vera: analysis of a single center cohort of 2480 red cell masses. Haematologica. 2019 Aug 14. [Epub ahead of print]

46. Silver RT, Krichevsky S. Distinguishing essential thrombocythemia JAK2V617F from polycythemia vera: limitations of erythrocyte values. Haematologica.2019;104(11):2200-2205.

47. Gisslinger $\mathrm{H}$, Zagrijtschuk $\mathrm{O}$, Buxhofer-Ausch $\mathrm{V}$, et al. Ropeginterferon alfa- $2 b$, a novel IFN $\alpha-2 b$, induces high response rates with low toxicity in patients with polycythemia vera. Blood. 2015;126(15):1762-1769.

48. Hasselbalch HC, Holmström MO. Perspectives on interferon-alpha in the treatment of polycythemia vera and related myeloproliferative neoplasms: minimal residual disease and cure? Semin Immunopathol. 2019;41(1):5-19.

49. Silver RT, Gjoni S. The hematocrit value in polycythemia vera: caveat utilitor. Leuk Lymphoma. 2015;56(5):1540-1541.

50. Spivak JL. Polycythemia vera: myths, mechanisms, and management. Blood. 2002;100(13):4272-4290.

51. Pearson TC, Wetherley-Mein G. Vascular occlusive episodes and venous haematocrit in primary proliferative polycythaemia. Lancet. 1978;2(8102):1219-1222

52. Pearson TC. Hemorheologic considerations in the pathogenesis of vascular occlusive events in polycythemia vera. Semin Thromb Hemost. 1997;23(5):433-439.

53. Pearson TC, Guthrie DL, Simpson J, et al. Interpretation of measured red cell mass and plasma volume in adults: Expert Panel on Radionuclides of the International Council for Standardization in
Haematology. Br J Haematol. 1995;89(4):748-756.

54. Marchioli R, Finazzi G, Specchia G, et al. ; CYTO-PV Collaborative Group. Cardiovascular events and intensity of treatment in polycythemia vera. N Engl J Med. 2013;368(1):22-33

55. Warny M, Helby J, Birgens HS, et al. Arterial and venous thrombosis by high platelet count and high hematocrit: 108521 individuals from the Copenhagen General Population Study. J Thromb Haemost. 2019 Jul 15. [Epub ahead of print]

56. Kannel WB, Gordon T, Wolf PA, McNamara P. Hemoglobin and the risk of cerebral infarction: the Framingham study. Stroke. 1972;3(4):409-420.

57. Pedersen KM, Bak M, Sørensen AL, et al. Smoking is associated with increased risk of myeloproliferative neoplasms: A general population-based cohort study. Cancer Med. 2018;7(11):5796-5802.

58. Barosi G, Buratti A, Costa A, et al. An atypical myeloproliferative disorder with high thrombotic risk and slow disease progression. Cancer. 1991;68(10):2310-2318.

59. Osler W. A clinical lecture on erythremia. Lancet. 1908;171(1):143146.

60. Weber FP. Polycythaemia, erythrocytosis and erythraemia. Q J Med. 1908:2:85-134

61. Gordeuk VR, Key NS, Prchal JT. Re-evaluation of hematocrit as a determinant of thrombotic risk in erythrocytosis. Haematologica. 2019 Apr;104(4):653-8.)

62. Nersesjan V, Zervides KA, Sørensen AL, Kjær L, Skov V, Hasselbalch $\mathrm{HC}$. The red blood cell count and the erythrocyte sedimentation rate in the diagnosis of polycythemia vera. Eur J Haematol 2019 Accepted for publication.

\title{
Standing at odds: mutated RAS and hematopoietic stem cells
}

\author{
Monica Nafria and Constanze Bonifer \\ Institute of Cancer and Genomic Sciences, College of Medicine and Dentistry, University of Birmingham, Birmingham, UK \\ E-mail: CONSTANZE BONIFER - c.bonifer@bham.ac.uk
}

doi:10.3324/haematol.2019.230029

A cute myeloid leukemia (AML) is the most common acute leukemia in adults and is characterized by the accumulation of myeloid leukemic blasts unable to complete differentiation. However, AML is a complex disease with variable outcomes and prognoses. ${ }^{1}$ Underlying these heterogeneous phenotypes is the fact that each sub-type of AML is defined by a different set of mutations and is controlled by a specific transcriptional and signaling network distinct to that of normal stem and progenitor cells. ${ }^{2}$ Genes mutated in AML are involved in gene regulation and include transcription factors, chromatin modifiers / remodelers, splicing regulators, DNA methyltransferases and signaling regulators that control the activity of inducible transcription factors. The result is a profound deviation from the normal differentiation trajectory, with each AML sub-type taking a different path and establishing its own cellular identity. ${ }^{2,3}$ Most AML sub-types carry more than one mutation and, with the exception of MLL-translocations (which are a hallmark of pediatric $\mathrm{AML}^{4}$ ), for a number of sub-types it has been shown that the first oncogenic hit is not sufficient to cause overt leukemia. In AML patients, mutations in genes from different functional categories co-exist, and data from sequencing studies as well as mouse models support this notion. ${ }^{5,6}$

The $\mathrm{t}(8 ; 21)$ translocation, occurring in $7 \%$ of adult de novo patients, is one of the most frequent cytogenetic aberrations in AML. 'This translocation fuses the DNAbinding domain coding region of the hematopoietic master regulator RUNX1 (AML1) to the Eight-twenty-One (ETO, RUNX1T1 or MTG8) gene, which encodes a nuclear co-repressor. The result is the formation of the AML1-ETO (alternatively named RUNX1-ETO) chimeric protein, which retains the ability to bind to RUNX1 binding motifs but lacks the transactivation domain of RUNX1., Germline expression of full-length AML1-ETO in mice causes embryonic lethality, ${ }^{10,11}$ but conditional expression in hematopoietic stem cells (HSC) leads to an initial expansion of myeloid progenitor cells, including HSC and granulocyte-macrophage progenitors (GMP). Such expansion was also seen with AML1-ETO-transduced human cord blood-derived HSC in vitro. ${ }^{12}$ Fusion $\mathrm{t}(8 ; 21)$ transcripts have been detected in utero and in postnatal blood samples ${ }^{13}$ and remain expressed at low levels in blood samples from t(8;21) AML patients in long-term remission. ${ }^{14}$ Furthermore, several AML1-ETO-expressing mouse models have failed to fully develop t(8;21) AML unless challenged by mutagenesis or aging, ${ }^{15-18}$ indicating the necessity of additional secondary mutations. These findings suggest that this chromosomal rearrangement is the driver mutation establishing a pre-leukemic clone. This notion is supported by the finding that $t(8 ; 21)$ patients present with a number of different secondary mutations. ${ }^{19}$ The most prominent of these mutations 